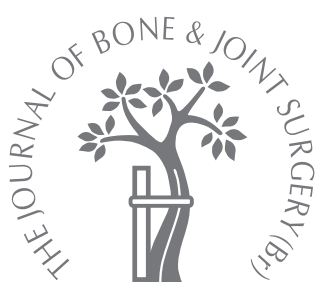

J. P. Cobb, H. Dixon, W. Dandachli, F. Iranpour

From Charing Cross Hospital, London, England

\title{
The anatomical tibial axis
}

\author{
RELIABLE ROTATIONAL ORIENTATION IN KNEE REPLACEMENT
}

The rotational alignment of the tibia is an unresolved issue in knee replacement. A poor functional outcome may be due to malrotation of the tibial component. Our aim was to find a reliable method for positioning the tibial component in knee replacement.

CT scans of 19 knees were reconstructed in three dimensions and orientated vertically. An axial plane was identified $20 \mathrm{~mm}$ below the tibial spines. The centre of each tibial condyle was calculated from ten points taken round the condylar cortex. The tibial tubercle centre was also generated as the centre of the circle which best fitted eight points on the outside of the tubercle in an axial plane at the level of its most prominent point.

The derived points were identified by three observers with errors of $0.6 \mathrm{~mm}$ to $1 \mathrm{~mm}$. The medial and lateral tibial centres were constant features (radius $24 \mathrm{~mm}$ (SD 3), and $22 \mathrm{~mm}$ (SD 3), respectively). An anatomical axis was created perpendicular to the line joining these two points. The tubercle centre was found to be $20 \mathrm{~mm}$ (SD 7) lateral to the centre of the medial tibial condyle. Compared with this axis, an axis perpendicular to the posterior condylar axis was internally rotated by $6^{\circ}$ (SD 3). An axis based on the tibial tubercle and the tibial spines was also internally rotated by $5^{\circ}$ (SD 10$)$.

Alignment of the knee when based on this anatomical axis was more reliable than either the posterior surfaces or any axis involving the tubercle which was the least reliable landmark in the region.

Numerous studies have shown that total knee replacement is a successful procedure, ${ }^{1-6}$ but it remains an operation with a considerable rate of failure. ${ }^{7}$ Discrepancies in reported results may be related to differences in outcome measurement. That noted, a substantial rate of dissatisfaction is observed if results are judged by reliable scoring systems that differentiate between successful and unsuccessful procedures. ${ }^{8,9}$ One of the reasons for dissatisfaction or failure may be malpositioning of the components of the knee replacement. ${ }^{10}$

In order to communicate effectively about alignment or positioning of components in knee replacement, a shared vocabulary is essential. A common frame of reference is required by investigators to describe accurately a bone or an anatomical feature and its orientation. This should have three axes and a point of origin. Without such a frame of reference, accurate surgery is difficult, since the surgeon cannot reliably describe the ideal position of any feature or component. In the hip, the anterior pelvic plane is now accepted as the functional frame of reference in relation to which the acetabular position can be described, ${ }^{11,12}$ although other reference frames have also been described which may have practical advantages. ${ }^{13}$ In the knee, the rotational alignment of the distal femur remains controversial, with Whiteside's line being used by some as a reference, ${ }^{14,15}$ despite the fact that it shows considerable variation even in normal subjects. ${ }^{14}$ Others advocate the use of the epicondylar axis ${ }^{16}$ although the reliability of this as a working frame of reference has been questioned. ${ }^{17-19}$ The posterior condylar axis is also used based on a line joining the most posterior surfaces of the femoral condyles. It is easily identifiable and is derived from the measurement of the most posterior surfaces of the femoral condyles. ${ }^{16}$ However, in pathological anatomy with a smaller than average lateral femoral condyle, its use will give rotational malalignment. ${ }^{20}$

There has been no universally-accepted tibial frame of reference. While there is agreement regarding the definition of normal coronal (varus/valgus) alignment and posterior tibial slope ${ }^{21}$ no consensus has been reached about the normal rotational axis of the tibia. Thus all studies relating knee function to the position or alignment of a component may be compro- 
mised by a failure to identify the sagittal plane of the tibia in a reliable way.

Rotational variation is a key determinant of function and of predisposition to disease. ${ }^{22-24}$ This is hard to measure from plain radiographs, although from short-leg radiographs of the knee alone, a standard $6^{\circ}$ of valgus for the femur has been reported as being acceptable for practical purposes. ${ }^{25}$ In a dry-bone study, the influence of undetected rotation of the knee was shown to change the surgeon's perception of alignment substantially. A neutral transverse tibial cut with a $10^{\circ}$ posterior slope was reported as varying from $5^{\circ}$ varus to $3^{\circ}$ of valgus with rotation of the tibia. ${ }^{26}$ Rotation is reliably measured using CT. ${ }^{27}$ Medial compartment osteoarthritis has been linked to reduced tibial external torsion, while patellofemoral osteoarthritis has been related to increased tibial external torsion. ${ }^{22-24}$ In a study of normal and osteoarthritic patients, the sagittal axis of the tibia as defined by the tibial tubercle was found to vary by over $40^{\circ}$ in respect to both the ankle and the epicondylar axis of the femur. ${ }^{28}$ In another study the angle between the sagittal axis of the knee and the ankle was variable with a range of over $16^{\circ} .^{29}$

Rotation of the tibial component has also been recognised as an important factor in the outcome of knee replacement. ${ }^{10}$ In a review of rotation of knee replacements based on CT findings, there was a range of rotation of the tibial components of $25^{\circ}$ compared with $9^{\circ}$ for rotation of the femoral components. ${ }^{30}$ In another study of 109 knees, 50\% were found to have tibial malrotation of more than $5^{\circ} .{ }^{31}$

Several authors have attempted to define a sagittal axis which would identify rotation using anatomical landmarks. Most proximally, the tibial sagittal plane has been derived from the position of the patella. ${ }^{32}$ This will be altered by anatomy unrelated to the tibia, the position of the joint, pathology of the joint and biomechanics. It is therefore unreliable.

Using landmarks within the knee or close to it, various sagittal planes have been described including a line from the mid-point between the tibial spines, passing $1 \mathrm{~mm}$ medial to the medial border of the tubercle, ${ }^{33}$ a line perpendicular to the posterior joint surface passing through the medial third of the tibial tubercle, ${ }^{31,34}$ and a line passing through the middle of the posterior cruciate ligament and perpendicular to the projected femoral transepicondylar axis. ${ }^{30,35}$

Distal to the knee, a sagittal axis has also been described through the second metatarsal or through the middle of the ankle. ${ }^{29}$

None of these methods has been universally adopted. To do so, any sagittal axis has to fulfil two criteria. It must be reliably identifiable and it must be an accurate and true representation of the sagittal axis. Our aim, therefore, was to identify local landmarks in the knee which could be used in both health and disease to describe the sagittal axis of the tibia to define its rotational alignment. The secondary questions which arose were related to the shape of the tibial components. We attempted to ascertain if there was an optimal way of orientating tibial components, whether asymmetrical and symmetrical tibial components should be inserted in the same way and how the tibial components of unicompartmental knees should be orientated.

\section{Patients and Methods}

We analysed the CT scans of 19 knees from 19 patients (10 men and nine women) with a mean age of 54 years (29 to 77). They had been obtained using the Imperial protocol $^{36}$ from patients who had given their consent for use of the datasets in research. Of these patients 11 had undergone unilateral knee surgery and therefore the contralateral knees were studied. For each of the other eight patients, only one knee was analysed. Customised software was created and used for analysis of the CT datasets.

Identification of bony landmarks for the initial orientation. Conventional bony landmarks used to identify the sagittal plane at knee surgery were identified first. These included the centre of the ankle, the centre of the proximal tibia and the tibial tubercle (Fig. 1). The centre of the proximal tibia was approximated using the coronal, sagittal and axial sections at the level of the articular surface.

One data point was placed at the back of each tibial condyle close to the surface of the joint to define the posterior condylar points, and on the highest point of the medial and lateral tibial spines. The femur was then subtracted. All the data points were identified on the cortical bone. The tibia was orientated by aligning the line joining the centre of the talus and proximal tibial centre vertically and rotating $90^{\circ}$ along a horizontal axis to get a craniocaudal view of the tibial plateau. This initial orientation allowed collection of further data points in a standardised method from the entire series of bones, despite their differing morphology (Fig. 2).

Secondary orientation using derived data points. Three derived points were obtained based on data points taken from the surface of the tibia as follows:

The tibial tubercle centre. More than six data points were placed on the surface of the tubercle at the level of its most anterior point. The centre of the best-fit circle formed using these points was termed the tibial tubercle centre (Fig. 3). The lateral condylar centre. More than ten data points were placed around the edge of the cortex of the lateral condyle at the level of the articular surface. This surface was in good condition since the knees studied had medial compartment osteoarthritis. The root-mean-square of the error for the best-fit circle was calculated and the centre of that circle was termed the lateral condylar centre (Fig. 3).

The medial condylar centre. The medial tibial plateau was not as circular as the lateral plateau, but it became circular within a few millimetres of the joint surface. More than ten data points were taken $20 \mathrm{~mm}$ below the tip of the medial tibial spine. The highest point on the medial tibial spine could be easily identified radiologically and could also be digitised using a digitising probe. The root-mean-square of the error for the best-fit circle was calculated, and the centre of that circle was termed the medial condylar centre (Fig. 3). 


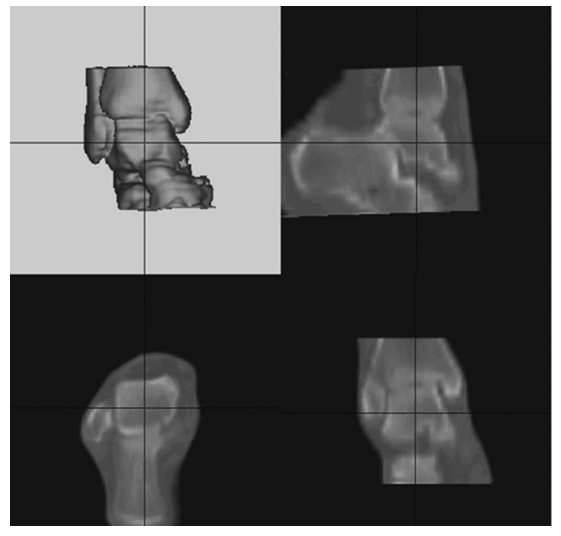

Fig. 1a

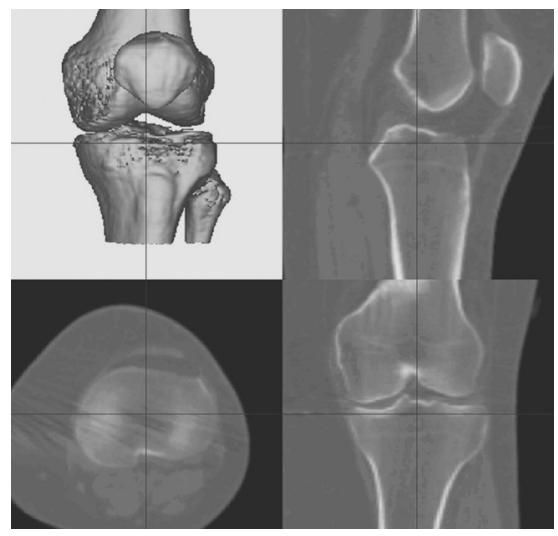

Fig. 1b

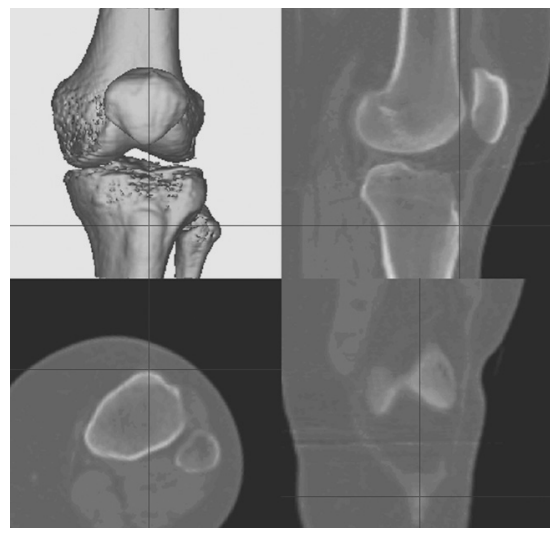

Fig. 1c

Tri-planar and 3D reconstruction views from the CT scans showing a) the centre of the ankle, with the plafond above it, b) the approximate centre of the proximal tibia, and c) the tip of the tibial tubercle.

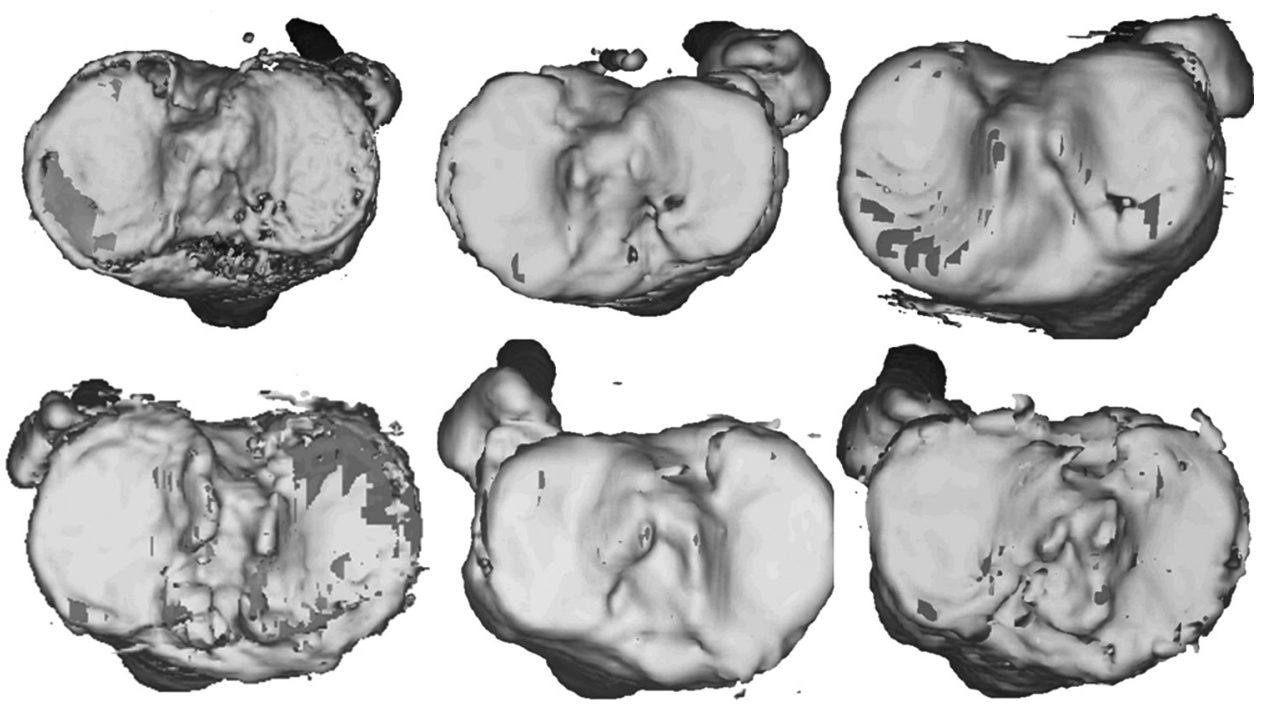

Fig. 2

Three-dimensional CT reconstruction of six of the tibiae showing the differing morphology and the variable position of the tibial tubercle relative to the tibial spines.

Sagittal axes. Three different sagittal axes were then created as follows:

The posterior condylar axis $\left(A_{P C}\right)$. This was defined as the perpendicular to the posterior condylar line at its midpoint. The two points defining the posterior condylar axis were the most prominent points posteriorly after the tibia had been orientated in the standard way. The posterior cortex of the tibia is used in several instrumentation systems in total knee replacement as a reference for positioning the tibial components. The sagittal axis is defined as being orthogonal to the $\mathrm{A}_{\mathrm{PC}}$.
The sagittal tubercle axis $\left(A_{S T}\right)$. A sagittal line was generated joining the lateral tibial spine and the tibial tubercle centre. A sagittal axis passing through the mid-point of the tibial attachment of the posterior cruciate ligament and the medial third of the patella tendon has been described commonly in the literature. ${ }^{35}$ We thought this axis was unreliable since the position of the patellar tendon deviated depending on the position of the limb. We therefore chose to use a sagittal axis which was lateral to this line with clearly identifiable data points, the tip of the lateral tibial spine and the tibial tubercle centre. We believe that the ori- 


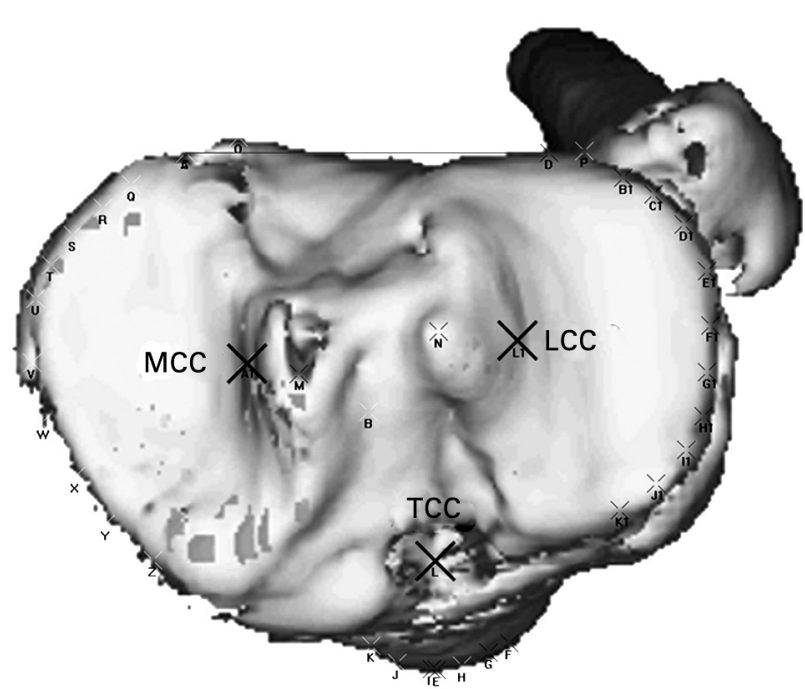

Fig. 3

CT reconstruction showing the medial (MCC) and lateral (LCC) condylar centres and the tibial tubercle centre (TTC).

entation of our line $\mathrm{A}_{\mathrm{ST}}$, was similar to the sagittal axis using the medial third of the tendon since both the anterior and posterior data points were based on similar landmarks. The anatomical tibial axis $\left(A_{T A}\right)$. This is the perpendicular at the mid-point of the line joining the medial and the lateral condylar centres. This axis is more independent of the relative change in size of the lateral condyle and of lateralisation of the tubercle (Fig. 4).

Variability and reliability. The anatomical and derived points, the axes and the dimensions were collected in 19 knees. Intra-observer repeatability was measured by repeating the data collection after one week. Two independent observers (WD, FI) repeated the entire process, from point gathering to measurements of angles. The reproducibility of this method was then calculated by using Bland-Altman analysis for interobserver agreement.

\section{Results}

Reliability of identification of derived landmarks. The tibial tubercle points fitted a circle with a mean root-meansquared error of $0.2 \mathrm{~mm}$ (SD 0.1). Points taken on the medial condylar cortex fitted a circle with a mean rootmean-squared error of $0.5 \mathrm{~mm}$ (SD 0.1). The lateral condylar cortex points fitted a circle with a mean root-meansquared error of $0.6 \mathrm{~mm}$ (SD 0.1). These derived landmarks, as centres of the circles, appeared to be reliably described by this method.

Relationship between the sagittal axes. The $A_{P C}$ was internally rotated in relation to the $A_{\mathrm{TA}}$ by a mean of $6^{\circ}$ (SD 3), reflecting the difference in radius of the two tibial condyles. A smaller lateral tibial condyle had the effect of relatively

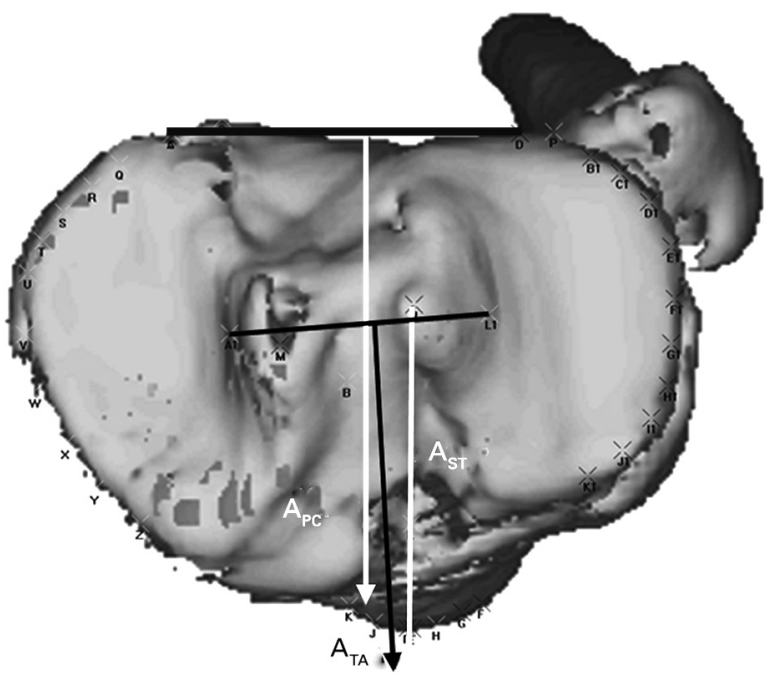

Fig. 4

$C T$ reconstruction showing the three sagittal axes on a single tibia. $A_{P C}$ posterior condylar axis (internally rotated $6^{\circ}(S D 3) ; A_{T A}$ anatomical tibia axis; $A_{S T}$, sagittal tubercle axis (internally rotated $5^{\circ}$ (SD 10)).

internally rotating the $\mathrm{A}_{\mathrm{PC}}$, because the posterior condylar line internally rotates relative to the sagittal plane. This is not expected to occur with the $\mathrm{A}_{\mathrm{TA}}$ as the centre of the condyle is not expected to change.

The $\mathrm{A}_{\mathrm{ST}}$ was internally rotated on the $\mathrm{A}_{\mathrm{TA}}$ by a mean of $5^{\circ}$ (SD 10). The SD reflected the variability of the position of the tubercle (Fig. 4).

Reliability of derived landmarks. The interobserver measurements were made on the same datasets, and comparison was made between observers. The $\mathrm{x}$-axis was mediolateral, the $y$-axis superoinferior, and the $\mathrm{z}$-axis anteroposterior. Analysis of the variation between observers 1 and 2 showed that the lateral condylar centre was slightly more reliably identified in the anteroposterior dimension than the medial condylar centre (mean difference $0.6 \mathrm{~mm} v s 1.2 \mathrm{~mm}$ ), while both were equally identified in the mediolateral dimension (mean difference $1.3 \mathrm{~mm}$ for both) (Fig. 5).

The tibial anatomical reference frame. Having established the relationship amongst the sagittal axes, the $\mathrm{A}_{\mathrm{TA}}$ as being the most reliable of the three sagittal axes, the dimensions of the knees were then measured and the relationship between the different morphological features described. Using the $\mathrm{A}_{\mathrm{TA}}$ and the longitudinal anatomical axis of the tibia to adjust rotation in the axial and sagittal planes, respectively, a frame of reference could be defined with the $\mathrm{x}$-axis being mediolateral, the $\mathrm{y}$-axis superoinferior, and the $\mathrm{z}$-axis anteroposterior.

A morphometric analysis of the proximal tibia was then performed. The mean medial tibial condyle radius was $24 \mathrm{~mm}$ (SD 3) and the mean lateral tibial condyle radius 


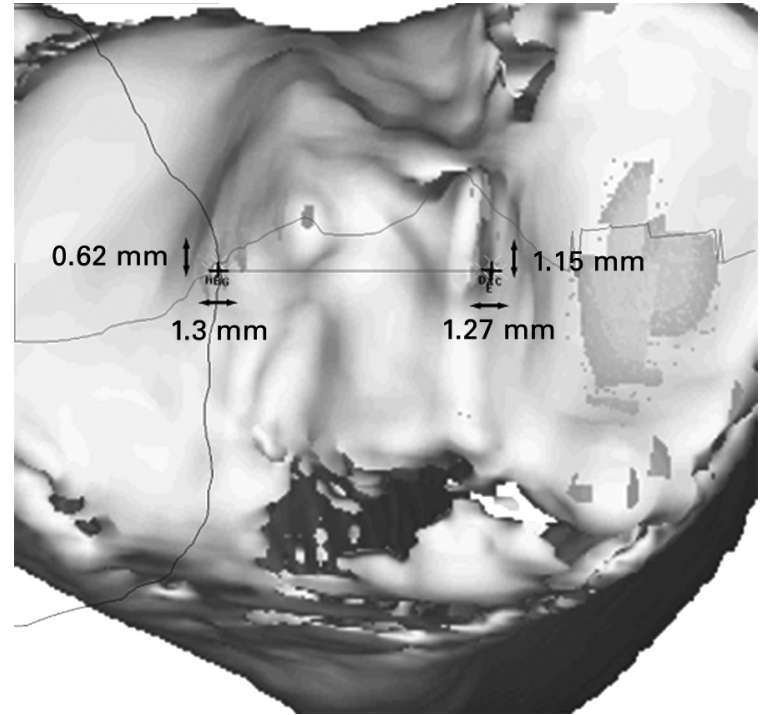

Fig. 5

CT scan showing the variability in identification of the condylar centres expressed as the size of the mean difference between observers 1 and 2 .

$22 \mathrm{~mm}$ (SD 3). The mean distance between the medial and lateral condylar centres was $26 \mathrm{~mm}$ (SD 6). The mean anterior distance from the line joining the two condylar centres to the tibial tubercle centre was $23 \mathrm{~mm}$ (SD 5). The tibial tubercle centre was located $20 \mathrm{~mm}$ (SD 7) lateral to the medial condylar centre. The y-axis scale was defined by the perpendicular distance from the lateral condylar centre to the tibial tubercle centre at $24 \mathrm{~mm}$ (SD 8) (Fig. 6). The position of the tibial tubercle centre had the largest variation amongst the knees studied. This suggests that it is not an ideal feature on which to base a frame of reference.

Intra- and inter-observer investigations. All of the interobserver repeated observations were within a range of $3.2^{\circ}$. The comparison of measurements of the posterior condylar line were within $1^{\circ}$. The intraclass correlation coefficient was 0.94 suggesting good agreement. The intra-observer repeatability after a delay of one week revealed a mean difference in all the projected angles of less than $0.5^{\circ}$.

\section{Discussion}

Our study had limitations. First, the number of knees studied was small. This may have been the reason behind the variations across the sample. This small sample may not be representative of the population. Another limitation was that the knees were arthritic rather than normal. However, the areas where the landmark points were chosen were disease-free. The method appeared to be reasonably reliable showing that we could establish a rotational axis of the tibia. The reliability and repeatability studies confirmed

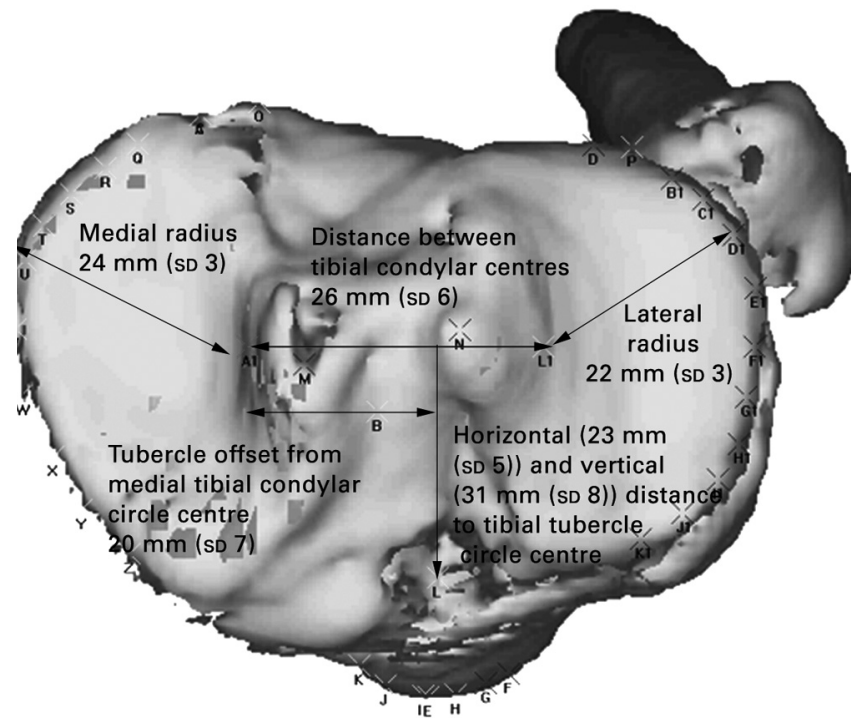

Fig. 6

$\mathrm{CT}$ reconstruction showing the relative sizes of the different components of the tibial plateau.

this since the $\mathrm{A}_{\mathrm{TA}}$ generated by each of the three observers was within $3^{\circ}$.

Low-dose CT scans have a radiation dose equivalent to long-leg radiographs. It is possible to obtain 3-D data without excessive ionising radiation. CT does incur extra financial cost and it is more time-consuming to scan the knee and analyse the data, but it does provide valuable information especially if complex procedures are being considered.

This method allowed us to identify landmarks in the knee and to describe their relationships to each other. The geometrical centre of the medial compartment was the most constant landmark in these knees. The lateral compartment had a geometrical centre which could also be derived from points on the cortex. It was equally constant in the mediolateral dimension, but varied more than that in the anteroposterior dimension. The tibial tubercle centre was also reliably derived by this method with small interobserver errors. However, the tubercle varied in position more than any other point in the mediolateral plane. Thus, any axis defined by the tubercle would also vary substantially.

The $\mathrm{A}_{\mathrm{TA}}$ sagittal axis varied depending on the differing dimensions of the medial and lateral condyles and was independent of the position of the tubercle. As the size of the lateral condyle increased the axis internally rotated. This variation was substantially less than that seen when the posterior condylar axis was used. The posterior condylar axis varied in its rotational alignment by approximately twice the $\mathrm{A}_{\mathrm{TA}}$ axis.

The tibial tubercle was the landmark which varied more than any other in the proximal tibia. While it is closely related to the position and morphology of the patello- 
Table I. The impact of the sagittal axis chosen for alignment of different tibial components

\begin{tabular}{|c|c|c|c|}
\hline & Posterior condylar axis $\left(A_{P C}\right)$ & Sagittal tubercle axis $\left(A_{S T}\right)$ & Anatomical tibial axis $\left(A_{T A}\right)$ \\
\hline \multicolumn{4}{|c|}{ Total knee replacement } \\
\hline Symmetrical & $\begin{array}{l}\text { Internal rotation of component } \\
\text { with asymmetrical tibia }\end{array}$ & $\begin{array}{l}\text { Excessive external rotation with } \\
\text { lateral tubercle }\end{array}$ & $\begin{array}{l}\text { Rotation appropriate to the } \\
\text { individual }\end{array}$ \\
\hline Asymmetrical & $\begin{array}{l}\text { External rotation of component } \\
\text { with symmetrical tibia }\end{array}$ & $\begin{array}{l}\text { Excessive internal rotation with } \\
\text { medial tubercle }\end{array}$ & $\begin{array}{l}\text { Rotation appropriate to the } \\
\text { individual }\end{array}$ \\
\hline \multicolumn{4}{|c|}{$\begin{array}{l}\text { Unicompartmental knee } \\
\text { replacement }\end{array}$} \\
\hline Medial & Not practical & $\begin{array}{l}\text { Variably externally rotated } \\
\text { component causing impingement }\end{array}$ & $\begin{array}{l}\text { Rotation appropriate to the } \\
\text { individual }\end{array}$ \\
\hline Lateral & Not practical & $\begin{array}{l}\text { Variably internally rotated } \\
\text { component causing impingement }\end{array}$ & $\begin{array}{l}\text { Rotation appropriate to the } \\
\text { individual }\end{array}$ \\
\hline
\end{tabular}

femoral joint, ${ }^{37}$ when the tibial sagittal axis was based on its position, there was substantial variation in alignment in relation to the articular surfaces.

This way of looking at the proximal tibia may be used to help to optimise the orientation of an arthroplasty in a knee with abnormally orientated landmarks. The practical application is when using a device with a posterior slope and our recommendation is that the axis be estimated from the relative size of the medial and lateral compartments before the cut. The condylar centre can be found by halving the anteroposterior dimension in each compartment. This can be fine-tuned after the bony section. We performed the analysis on virtual cuts at a depth of about $8 \mathrm{~mm}$ below the joint line and found that the cross-section of the condyles was more circular at that level than at the articular surface where the extension facet of the medial compartment was not spherical. When using surgical navigation, the algorithm could be best used to help to define rotational orientation during the bone morphing stage. This method is clearly ideal for use with a CT-based pre-operative planning system, but currently very few surgeons have access to such technology. Detailed pre-operative planning continues to have proponents. If there is a method for identifying the rotational alignment with greater confidence, there may be more demand for it.

Our morphological study cannot directly answer the important question as to whether there is an optimal orientation of the tibial component of a total or partial knee replacement. It does, however, provide a reproducible way of orientating the tibia and a basis from which to plan surgery. With it, both investigators and surgeons will be able to plan surgery and report on the functional outcome afterwards. They will also be able to measure and report the rotation of any inserted component in a reliable way. Without it, there is a risk that any report of a series of operations will be confounded by surgical bias or errors, both of which go unrecognised. Surgeons who prefer to orientate the tibial component to optimise patellar tracking may compromise tibiofemoral articulation. Their method of orientation based on the tubercle has attractions in terms of tracking, but risks malorientation in either internal or external rota- tion since the tubercle is an independent variable with a substantial range. By basing tibial orientation on the posterior tibial condyles, surgeons will risk malorientation which will be greatest when the difference in size of the condyles is greatest. There is as yet no method of dissociating the patellofemoral joint from the tibiofemoral joint in total knee replacement.

When inserting specific tibial components, there are implications for the knee replacement surgeon which are summarised in Table I.

The use of an axis which is based on the relative sizes of each tibial compartment will influence the orientation of the tibial component in symmetrical total knee replacements. When the condyles are roughly the same size, the posterior condylar axis provides a more reliable method of orientation than any tubercle-based method. As the difference in the size of the condyles increases, the use of a symmetrical component becomes more problematic. The use of the posterior condylar axis will rotate a component relatively internally leading to prominence of the component on the lateral side. By downsizing the tibial component in these circumstances, the component will then be relatively undersized on the medial side.

The use of asymmetrical tibial components produces the opposite problem. A knee with nearly symmetrical condyles orientated using the posterior condylar axis will rotate the tibial component externally while extremes of asymmetry will lead to internal rotation of the component.

Medial unicompartmental knee replacements with a mobile bearing have a sagittal axis which is an important part of their function. Malalignment in this axis will predispose to maltracking and can contribute to bearing dislocation. The use of the anatomical tibial axis as part of preoperative planning will minimise this, while basing alignment on the tibial tubercle will in some cases lead to the tibial component being considerably externally rotated, leading to meniscal bearing impingement in flexion.

Lateral unicompartmental knee replacement is not in common use, with difficulty in orientation of the tibial component often cited as one of the reasons for failure of the device. The use of any axis based on the tibial tubercle 
for all patients will cause a variation in the degree of external rotation of the tibial component. A sagittal axis based on the patient's own anatomy may be a safer method of avoiding excessive internal rotation causing posterior impingement or external rotation resulting in anterior impingement.

In summary, tibial geometry has long been a problem for all surgeons aspiring to undertake accurate surgery. Our study has provided a means for allowing the surgeon to describe the tibial plateau and to orientate the surgery in a reliable way. Only when components are orientated accurately will it be possible to ascertain how successful any one device is. Until then, we may simply be documenting the extent to which a particular device or patient is forgiving of variation in rotational alignment.

\section{Supplementary Material}

[ё A table showing the geometrical results for the proximal tibia, measured by the three observers is available with the electronic version of this article on our website at www.jbjs.org.uk

We would like to thank $\mathrm{Dr}$ R. Richards for his support with the software. No benefits in any form have been received or will be received from a commercial party related directly or indirectly to the subject of this article.

\section{References}

1. Gill GS, Joshi AB, Mills DM. Total condylar knee arthroplasty: 16- to 21-year results. Clin Orthop 1999;367:210-15.

2. Ranawat CS, Flynn WF Jr, Saddler S, Hansraj KK, Maynard MJ. Long-term results of the total condylar knee arthroplasty: a 15-year survivorship study. Clin Orthop 1993;286:94-102.

3. Epinette JA, Manley MT. Hydroxyapatite-coated total knee replacement: clinical experience at 10 to 15 years. J Bone Joint Surg [Br] 2007;89-B:34-8.

4. Baker PN, Khaw FM, Kirk LMG, Esler CNA, Gregg PJ. A randomised controlled trial of cemented versus cementless press-fit condylar total knee replacement: 15year survival analysis. J Bone Joint Surg [Br] 2007;89-B:1608-14.

5. Tai CC, Cross MJ. Five- to 12-year follow-up of a hydroxyapatite-coated, cementless total knee replacement in young, active patients. J Bone Joint Surg [Br] 2006;88B:1158-63.

6. Roberts VI, Esler CNA, Harper WM. A 15-year follow-up study of 4606 primary total knee replacements. J Bone Joint Surg [Br] 2007;89-B:1452-6.

7. Kurtz S, Ong K, Lau E, Mowat F, Halpern M. Projections of primary and revision hip and knee arthroplasty in the United States from 2005 to 2030. J Bone Joint Surg [Am] 2007;89-A:780-5.

8. Gregg P, et al. National Joint Registry for England and Wales 2 nd annual report. http://www.njircentre.org.uk/documents/reports/annual/2nd/NJR2_fullreport.pdf (date last accessed 16 June 2008).

9. Weiss JM, Noble PC, Conditt MA, et al. What functional activities are important to patients with knee replacements? Clin Orthop 2002;404:172-88.

10. Windsor RE, Scuderi GR, Moran MC, Insall JN. Mechanisms of failure of the femoral and tibial components in total knee arthroplasty. Clin Orthop 1989;248:15-19.

11. Jaramaz B, DiGioia AM 3rd, Blackwell M, Nikou C. Computed assisted measurement of cup placement in total hip replacement. Clin Orthop 1998;354:70-81.

12. Blendea S, Eckman K, Jaramaz B, Levison TJ, DiGioia AM 3rd. Measurements of acetabular cup position and pelvic spatial orientation after total hip arthroplasty using computed tomography/radiography matching. Comput Aided Surg 2005;10:37 43

13. Dandachli W, Richards R, Sauret V, Cobb JP. The transverse pelvic plane: a new and practical reference frame for hip arthroplasty. Comput Aided Surg 2006;11:322-6.

14. Middleton FR, Palmer SH. How accurate is Whiteside's line as a reference axis in total knee arthroplasty? Knee 2007;14:204-7.

15. Picard F, Gregori A, Dean F, Mennessier A, Dillon J. Computer-assisted dynamic total knee arthroplasty using Whiteside's line for alignment. Orthopedics 2006;29(Suppl):104-7.

16. Nagamine R, Miura H, Inoue Y, et al. Reliability of the anteroposterior axis and the posterior condylar axis for determining rotational alignment of the femoral component in total knee arthroplasty. J Orthop Sci 1998;3:194-8.

17. Coughlin KM, Incavo SJ, Churchill DL, Beynnon BD. Tibial axis and patellar position relative to the femoral epicondylar axis during squatting. J Arthroplasty 2003;18:1048-55

18. Kinzel VM, Ledger M, Shakespeare $\mathbf{D}$. Can the epicondylar axis be defined accurately in total knee arthroplasty? Knee 2005;12:293-6.

19. Tanavalee A, Yuktanandana P, Ngarmukos C. Surgical epicondylar axis vs anatomical epicondylar axis for rotational alignment of the femoral component in total knee arthroplasty. J Med Assoc Thai 2001;84(Suppl 1):401-8.

20. Newbern DG, Faris PM, Ritter MA, et al. A clinical comparison of patellar tracking using the transepicondylar axis and the posterior condylar axis. J Arthroplasty 2006;21:1141-6.

21. Yoo JH, Chang CB, Shin KS, Seong SC, Kim TK. Anatomical references to assess the posterior tibial slope in total knee arthroplasty: a comparison of 5 anatomical axes. J Arthroplasty 2008;23:586-92.

22. Takai S, Sakakida K, Yamashita F, Suzu F, Izuta F. Rotational alignment of the lower limb in osteoarthritis of the knee. Int Orthop 1985;9:209-15.

23. Cooke TD, Price N, Fisher B, Hedden D. The inwardly pointing knee: an unrecognized problem of external rotational malalignment. Clin Orthop 1990;260:56-60.

24. Eckhoff DG, Johnston RJ, Stamm ER, Kilcoyne RF, Wiedel JD. Version of the osteoarthritis knee. J Arthroplasty 1994;9:73-9.

25. Oswald MH, Jakob RP, Schneider E, Hoogewoud HM, et al. Radiological analysis of normal axial alignment of femur and tibia in view of total knee arthroplasty. $J$ Arthroplasty 1993:8:419-26.

26. Lonner JH, Laird MT, Stuchin SA. Effect of rotation and knee flexion on radiographic alignment in total knee arthroplasties. Clin Orthop 1996;331:102-6.

27. Jazrawi LM, Birdzell L, Kummer FJ, Di Cesare PE, et al. The accuracy of computed tomography for determining femoral and tibial total knee arthroplasty component rotation. J Arthroplasty 2000;15:761-6.

28. Nagamine R, Miyanishi K, Miura H, et al. Medial torsion of the tibia in Japanese patients with osteoarthritis of the knee. Clin Orthop 2003;408:218-24.

29. Mizu-uchi H, Matsuda S, Miura H, et al. The effect of ankle rotation on cutting of the tibia in total knee arthroplasty. J Bone Joint Surg [Am] 2006;88-A:2632-6.

30. Asano T, Akagi M, Koike K, Nakamura T. In vivo three-dimensional patellar tracking on the femur. Clin Orthop 2003;413:222-32.

31. Uehara K, Kadoya Y, Kobayashi A, Ohashi H, Yamano Y. Bone anatomy and rotational alignment in total knee arthroplasty. Clin Orthop 2002;402:196-201.

32. Minns RJ, Bibb R, Banks R, Sutton RA. The use of a reconstructed three-dimensional solid model form CT to aid the surgical management of a total knee arthroplasty: a case study. Med Eng Phys 2003;25:523-6.

33. Dalury DF. Observations of the proximal tibia in total knee arthroplasty. Clin Orthop 2001;389:150-5.

34. Luo CF. Reference axes for reconstruction of the knee. Knee 2004;11:251-7.

35. Akagi M, Oh M, Nonaka T, et al. An anteroposterior axis of the tibia for total knee arthroplasty. Clin Orthop 2004;420:213-19.

36. Henckel LJ, Richards R, Lozhkin K, et al. Very low-dose computed tomography for planning and outcome measurement in knee replacement: the imperial knee protocol. J Bone Joint Surg [Br] 2006;88-B:1513-18.

37. Yamada Y, Toritsuka Y, Horibe S, et al. In vivo movement analysis of the patella using a three-dimensional computer model. J Bone Joint Surg [Br] 2007;89 B:752-60. 